\title{
Care of patients with non-small-cell lung cancer stage III - the Central European real- world experience
}

\author{
Milada Zemanova', Robert Pirker², Lubos Petruzelka1, Zuzana Zbozínkova, \\ Dragana Jovanovic ${ }^{4}$, Mirjana Rajer ${ }^{5}$, Krisztina Bogos ${ }^{6}$, Gunta Purkalne ${ }^{7}$, Vesna Ceriman ${ }^{4}$, \\ Subhash Chaudhary ${ }^{8}$, Igor Richter ${ }^{9}$, Jirí Kufa ${ }^{10}$, Lenka Jakubikova ${ }^{11}$, Marius Zemaitis ${ }^{12}$, \\ Marketa Cernovska ${ }^{13}$, Leona Koubkova ${ }^{14}$, Zdenka Vilasova ${ }^{15}$, Karin Dieckmann ${ }^{16}$, \\ Attila Farkas ${ }^{17}$, Jelena Spasic ${ }^{18}$, Katerina Fröhlich ${ }^{3}$, Andreas Tiefenbacher, ${ }^{2}$ Virag Hollosi ${ }^{6}$, \\ Juraj Kultan ${ }^{10}$, Iveta Kolarová15, Jiri Votruba ${ }^{1}$
}

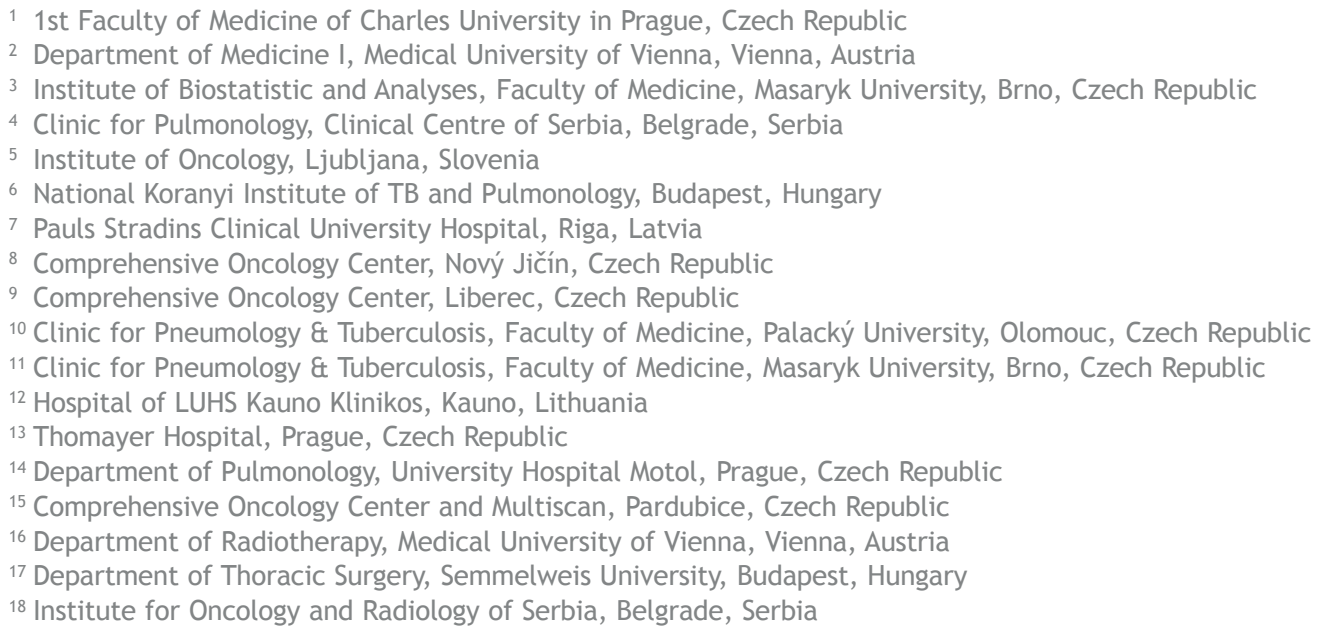

Background. Management of non-small-cell lung cancer (NSCLC) is affected by regional specificities. The present study aimed at determining diagnostic and therapeutic procedures including outcome of patients with NSCLC stage III in the realworld setting in Central European countries to define areas for improvements.

Patients and methods. This multicentre, prospective and non-interventional study collected data of patients with NSCLC stage III in a web-based registry and analysed them centrally.

Results. Between March 2014 and March 2017, patients ( $\mathrm{n}=583$ ) with the following characteristics were entered: $32 \%$ females, $7 \%$ never-smokers; ECOG performance status (PS) 0, 1, 2 and 3 in $25 \%, 58 \%, 12 \%$ and $5 \%$, respectively; $21 \%$ prior weight loss; $53 \%$ squamous carcinoma, $38 \%$ adenocarcinoma; $10 \%$ EGFR mutations. Staging procedures included chest X-ray $(97 \%$ of patients), chest CT (96\%), PET-CT (27\%), brain imaging (20\%), bronchoscopy (89\%), endobronchial ultrasound (EBUS) (13\%) and CT-guided biopsy (9\%). Stages IIIA/IIIB were diagnosed in 55\%/45\% of patients, respectively. N2/N3 nodes were diagnosed in $60 \% / 23 \%$ and pathologically confirmed in $29 \%$ of patients. Most patients $(56 \%)$ were treated by combined modalities. Surgery plus chemotherapy was administered to $20 \%$, definitive chemoradiotherapy to $34 \%$, chemotherapy only to $26 \%$, radiotherapy only to $12 \%$ and best supportive care (BSC) to $5 \%$ of patients. Median survival and progression-free survival times were 16.8 $(15.3 ; 18.5)$ and $11.2(10.2 ; 12.2)$ months, respectively. Stage IIIA, female gender, no weight loss, pathological mediastinal lymph node verification, surgery and combined modality therapy were associated with longer survival.

Conclusions. The real-world study demonstrated a broad heterogeneity in the management of stage III NSCLC in Central European countries and suggested to increase the rates of PET-CT imaging, brain imaging and invasive mediastinal staging.

Key words: diagnostic procedures; multimodality treatment; non-small-cell lung cancer; stage III 


\section{Introduction}

Management of locally advanced (stage III) nonsmall cell lung carcinoma (NSCLC) includes a broad spectrum of diagnostic procedures and therapeutic modalities. Diagnosis is primarily based on computer tomography (CT) scan of chest plus upper abdomen and brain, bronchoscopy and CT-guided biopsies. For more precise exclusion of distant metastases, the evaluation with PET-CT is useful. Detailed locoregional staging is done by means of PET-CT ${ }^{1}$, endobronchial or endoscopic ultrasound (EBUS/EUS), mediastinoscopy, thoracoscopy, mediastinotomy or other biopsies of suspected nodal lesions in order to distinguish early stages (stages I and II) from stage IIIA/IIIB. ${ }^{2}$

Treatment of NSCLC stage III requires multidisciplinary co-operation in order to deliver appropriate local and systemic therapies for the various subgroups. Occurrence of distant metastases with and without local progression is frequent, thereby leading to 5-year survival rates often less than $20 \%{ }^{3}$ According to the $7^{\text {th }}$ edition of the TNM classification $^{3}$ stage III was subdivided into stage IIIA and IIIB. The 5-year survival rates were $36 \%$ and $19 \%$, respectively. ${ }^{4}$ According to the $8^{\text {th }}$ edition, stage III is divided into IIIA, IIIB and IIIC. The 5-year survival rates are $36 \%, 26 \%$ and $13 \%$, respectively. ${ }^{4}$ In operable stage IIIA, induction or adjuvant chemotherapy improve overall survival ${ }^{5,6}$ and are established as standard treatments. Important prognostic factors associated with prolonged survival are pathological down-staging of mediastinal lymph nodes and/or primary tumors, and complete tumour resection. ${ }^{7-10}$ Some trials assessing induction therapy followed by surgery have also included patients with stage IIIB disease and suggested that patients with operable stage IIIB NSCLC have outcomes similar to those with stage IIIAN2 disease. ${ }^{11}$ For patients with inoperable stage IIIA or stage IIIB and good performance status (PS), definitive chemoradiotherapy is the treatment of choice. ${ }^{2}$ Concurrent chemoradiotherapy is associated with longer survival at increased toxicity compared to the sequential approach. ${ }^{12}$ Radiotherapy dose escalation has no clear benefit. ${ }^{13}$ Sequential chemoradiotherapy or radiotherapy alone remain options for selected patients. ${ }^{2}$ Consolidation therapy with durvalumab after concurrent chemoradiotherapy has recently been shown to improve survival of patients ${ }^{14}$ and has been established as standard treatment.

There is evidence that management of NSCLC stage III varies between countries, geographical regions, cancer centres and even treating physicians. Reasons for these variations include differences in regional standards, access to diagnostic procedures as well as therapeutic modalities, and resources. The aim of the present study was to determine diagnostic and therapeutic procedures as well as clinical outcome including survival of patients with NSCLC stage III in the real-world setting in Central European countries and to define areas for future improvements in routine management of these patients.

\section{Patients and methods}

\section{Patients}

The present study was prospective, observational, non-interventional, multicentric, multinational and registry based. The study had been approved by ethics committees of participating centres and was performed in accordance with the Declaration of Helsinki. Study entry criteria were patients of any age, histological and/or cytological diagnosis of NSCLC stage III according to $7^{\text {th }}$ edition of the TNM classification ${ }^{3}$ and signed written informed consent. The study allowed enrolling patients who have been treated between March 2014 and March 2017. Follow-up of patients continued until February 2018.

\section{Data collection}

Registration of all data was fully anonymous and performed in an electronic case report form (CRF [eCRF]) by qualified personnel. Patient identification was in the responsibility of each investigator. The following data were collected: age, gender, race, smoking status, PS, weight loss, date of NSCLC diagnosis, histology and mutational status of tumors, staging procedures, method of mediastinal lymph-node examination, TNM and tumor stage. Data collected on therapeutic procedures were primary therapy, surgical procedures (date, extent, completeness of resection, information on repeated resections), thoracic radiotherapy (date, dose, fractions, technique, energy), prophylactic cranial irradiation, and chemotherapy (dates of start and end, number of cycles, cytotoxic drugs). Combined modality therapy was assessed according to investigator statement and/or dates of overlapping therapies. Type of best treatment response, date of recurrence or progression, and dates of death were collected. 
TABLE 1. Patient characteristics

\begin{tabular}{|c|c|c|c|c|}
\hline \multirow{2}{*}{ Characteristic } & IIIA (N = 321) & IIIB $(N=262)$ & $I I I A+I I I B(N=583)$ & \multirow{2}{*}{$\mathrm{P} *$} \\
\hline & N (\%) & N (\%) & N (\%) & \\
\hline $\begin{array}{l}\text { Age } \\
<65 \text { years } \\
\geq 65 \text { years } \\
\text { Mean } \pm S D \text { (years) } \\
\text { Median (years) }\end{array}$ & $\begin{array}{l}155(48.3) \\
166(51.7) \\
64.8 \pm 10.5 \\
65.4\end{array}$ & $\begin{array}{l}117(44.7) \\
145(55.3) \\
65.1 \pm 10.1 \\
66.0\end{array}$ & $\begin{array}{c}272(46.7) \\
311(53.3) \\
64.9 \pm 10.3 \\
65.6\end{array}$ & 0.429 \\
\hline $\begin{array}{l}\text { Gender } \\
\text { Female } \\
\text { Male }\end{array}$ & $\begin{array}{c}92(28.7) \\
229(71.3)\end{array}$ & $\begin{array}{c}96(37.0) \\
166(63.6)\end{array}$ & $\begin{array}{l}188(32.2) \\
395(67.8)\end{array}$ & 0.050 \\
\hline $\begin{array}{l}\text { Smoking status } \\
\text { Current smoker } \\
\text { Former smoker } \\
\text { Never smoker } \\
\text { Unknown }\end{array}$ & $\begin{array}{l}161(50.2) \\
127(39.6) \\
26(8.1) \\
7(2.2)\end{array}$ & $\begin{array}{l}147(56.3) \\
92(35.2) \\
16(6.1) \\
7(2.7)\end{array}$ & $\begin{aligned} & 308(52.8) \\
& 219(37.6) \\
& 42(7.2) \\
& 14(2.4)\end{aligned}$ & 0.446 \\
\hline $\begin{array}{l}\text { Weight loss } \geq 10 \% \text { within prior } \\
\text { No } \\
\text { Yes } \\
\text { Unknown }\end{array}$ & $\begin{array}{c}243(75.7) \\
50(15.6) \\
28(8.7)\end{array}$ & $\begin{array}{l}163(62.5) \\
70(26.8) \\
29(11.0)\end{array}$ & $\begin{array}{c}406(69.6) \\
120(20.6) \\
57(9.8)\end{array}$ & $<0.001$ \\
\hline $\begin{array}{l}\text { WHO performance status } \\
0 \\
1 \\
2 \\
3\end{array}$ & $\begin{array}{l}86(26.8) \\
194(60.4) \\
31(9.7) \\
10(3.1)\end{array}$ & $\begin{array}{c}60(23.0) \\
144(54.9) \\
41(15.7) \\
17(6.5)\end{array}$ & $\begin{array}{l}146(25.0) \\
338(58.0) \\
72(12.3) \\
27(4.6)\end{array}$ & 0.025 \\
\hline $\begin{array}{l}\text { Histology } \\
\text { Squamous cell carcinoma } \\
\text { Adenocarcinoma } \\
\text { NSCLC NOS } \\
\text { Other }\end{array}$ & $\begin{array}{l}176(54.8) \\
115(35.8) \\
18(5.6) \\
12(3.7)\end{array}$ & $\begin{array}{l}133(51.0) \\
105(40.0) \\
18(6.9) \\
6(2.3)\end{array}$ & $\begin{array}{l}309(53.0) \\
220(37.7) \\
36(6.2) \\
18(3.1)\end{array}$ & 0.477 \\
\hline
\end{tabular}

* = Chi-square test for IIIA vs. IIIB; NOS = not otherwise specified; NSCLC = non-small-cell lung cancer; SD = standard deviation

\section{Statistical analyses}

Descriptive statistics and frequency tables were used to characterize the sample data set. Statistical significances of differences for categorical and continuous parameters were assessed by means of Fisher's exact test and Mann-Whitney test, respectively. Overall survival (OS) was defined as the time from treatment initiation until death of any cause. Progression-free survival (PFS) was defined as the time from treatment initiation until first documented progression and/or death of any cause. Patients without an event were censored at the time of last follow-up visit. Patients lost to follow-up were counted as interval-censored observations. The assumption was made that patients were lost to follow-up due to their treatment failure and that, therefore, no information is available about them. Interval set (interval between visits) was 6 months. ${ }^{15}$ PFS and OS were estimated by KaplanMeier analysis and 95\% confidence intervals (95\% CI) were provided for all point estimates. Statistical significance of differences in survival between the subgroups was assessed by means of the log-rank test. The multivariable Cox proportional hazard model was used to evaluate the effects of potential prognostic factors on survival measures. Hazard ratios (HR) were complemented with 95\% confidence intervals and supported with significance levels. Overall response rate (ORR) was defined as the sum of complete response rate (CR) and partial response rate $(\mathrm{PR})$ and disease control rate as ORR plus stable disease rate (SD). All statistical tests were performed at the significance level of $\mathrm{P}=0.05$.

\section{Results}

\section{Patients}

A total of 617 patients were enrolled but 34 patients were excluded due to various violations of the study protocol. Thus the study population comprises 583 patients from 16 centres of seven Central European countries: eight centres from Czech Republic (269 patients), two centres from Serbia (109 patients), two centres from Hungary (48 patients) and one centre each from Slovenia (53 patients), Latvia (43 patients), Lithuania (38 patients) and Austria (23 patients). The date for final analysis was February 19, 2018. Minimum followup since initial diagnosis was 11 months. Patient characteristics are shown in Table 1: 53.3\% aged $\geq$ 
TABLE 2. Diagnostic procedures

\begin{tabular}{lcc}
\hline \multirow{2}{*}{ Procedure } & \multicolumn{2}{c}{ Patients (N = 583) } \\
\cline { 2 - 3 } Chest CT scan & N (\%) & $\begin{array}{c}\text { range of \% } \\
\text { in centres }\end{array}$ \\
Chest X-ray & $567(97)$ & $67-100$ \\
Bronchoscopy & $559(96)$ & $79-100$ \\
Upper abdominal CT scan & $381(89)$ & $65-100$ \\
Upper abdominal US & $160(27)$ & $0-100$ \\
PET-CT or PET scan & $163(28)$ & $0-87$ \\
Brain CT or MRI & $117(20)$ & $0-91$ \\
Bone scan & $88(15)$ & $0-79$ \\
EBUS or EUS & $80(14)$ & $0-71$ \\
CT-guided biopsy & $54(9)$ & $0-31$ \\
VATS & $19(3)$ & $0-35$ \\
Mediastinoscopy & $13(2)$ & $0-13$ \\
Others & $47(8)$ & $0-30$ \\
\hline
\end{tabular}

EBUS = endobronchial ultrasound; EUS = endoscopic ultrasound; VATS = video-assisted thoracoscopic surgery
TABLE 3: TNM subgroups (Union for International Cancer Control [UICC] 7)

\begin{tabular}{|c|c|c|c|c|}
\hline \multirow{2}{*}{ TNM } & \multirow{2}{*}{$\begin{array}{c}\begin{array}{c}\text { Total patient } \\
\text { population }\end{array} \\
\mathrm{N}(\%) \\
\end{array}$} & \multicolumn{3}{|c|}{ Patients undergoing surgery } \\
\hline & & N & $\%$ of total population & $\%$ in stage subgroup \\
\hline$\| I I A+I I I B$ & $583(100)$ & 135 & 32.2 & NA \\
\hline IIIA & $321(55.1)$ & 119 & 20.4 & 37.1 \\
\hline $\begin{array}{l}\text { T4N0 } \\
\text { T3N1 } \\
\text { T4N1 } \\
\text { T1N2 } \\
\text { T2N2 } \\
\text { T3N2 }\end{array}$ & $\begin{array}{c}33(5.7) \\
39(6.7) \\
26(4.5) \\
25(4.3) \\
98(16.8) \\
100(17.2)\end{array}$ & $\begin{array}{l}10 \\
16 \\
7 \\
14 \\
45 \\
27\end{array}$ & $\begin{array}{l}1.7 \\
2.7 \\
1.2 \\
2.4 \\
7.7 \\
4.6\end{array}$ & $\begin{array}{l}30.3 \\
41.0 \\
26.9 \\
56.0 \\
45.9 \\
27.0\end{array}$ \\
\hline IIIB & $262(44.9)$ & 16 & 2.8 & 6.1 \\
\hline $\begin{array}{l}\text { T4N2 } \\
\text { T1N3 } \\
\text { T2N3 } \\
\text { T3N3 } \\
\text { T4N3 }\end{array}$ & $\begin{array}{c}129(22.2) \\
17(2.9) \\
32(5.5) \\
29(5.0) \\
55(9.4)\end{array}$ & $\begin{array}{l}15 \\
0 \\
1 \\
0 \\
0\end{array}$ & $\begin{array}{c}2.6 \\
0 \\
0.2 \\
0 \\
0\end{array}$ & $\begin{array}{c}11.6 \\
0 \\
3.1 \\
0 \\
0\end{array}$ \\
\hline \multicolumn{5}{|l|}{ T stage } \\
\hline $\begin{array}{l}\text { T1 } \\
\text { T2 } \\
\text { T3 } \\
\text { T4 }\end{array}$ & $\begin{array}{c}42(7.2) \\
130(22.3) \\
168(28.8) \\
243(41.7)\end{array}$ & $\begin{array}{l}14 \\
46 \\
43 \\
32\end{array}$ & $\begin{array}{l}2.4 \\
7.9 \\
7.4 \\
5.5\end{array}$ & $\begin{array}{l}33.3 \\
35.4 \\
25.6 \\
13.1\end{array}$ \\
\hline \multicolumn{5}{|l|}{$\mathrm{N}$ stage } \\
\hline $\begin{array}{l}\text { N0 } \\
\text { N1 } \\
\text { N2 } \\
\text { N3 }\end{array}$ & $\begin{array}{c}33(5.7) \\
65(11.1) \\
352(60.4) \\
133(22.8)\end{array}$ & $\begin{array}{c}10 \\
23 \\
101 \\
1\end{array}$ & $\begin{array}{l}1.7 \\
3.9 \\
17.3 \\
0.2\end{array}$ & $\begin{array}{c}30.3 \\
35.4 \\
28.7 \\
0.8\end{array}$ \\
\hline
\end{tabular}

NA $=$ not applicable
65 years, $32.2 \%$ females, $7.2 \%$ never-smokers, $20.6 \%$ weight loss ( $\geq 10 \%$ within prior three months), $83 \%$ PS WHO 0-1. Stages IIIA and IIIB were diagnosed in $321(55.1 \%)$ and $262(44.9 \%)$ patients, respectively. Females, patients with weight loss and patients with poor PS were slightly more frequent among patients with stage IIIB. Squamous cell carcinomas were diagnosed in 309 (53.0\%) patients, adenocarcinomas in $220(37.7 \%)$ patients, NSCLC not otherwise specified in $36(6.2 \%)$ patients, and other types (e.g. adeno-squamous, large cell) in 18 (3.1\%) patients. Results of molecular analyses were documented for 150 (25.7\%) patients (data not shown). EGFR mutations were detected in 14/142 (9.9\%) patients, KRAS mutations in $11 / 31$ (35.5\%) patients and ALK aberrations in 2/88 (2.3\%) patients (data not shown).

\section{Staging procedures}

Diagnostic and staging procedures are summarized in Table 2 . Widely used procedures were chest x-ray (96\%), chest CT scan $(97 \%)$, bronchoscopy $(89 \%)$ and upper abdominal CT scan (67\%). PET-CT or PET were performed in $28 \%$ of patients. Brain imaging by means of CT or MRI was done in $20 \%$ of patients. EBUS or EUS were performed in $14 \%$ of patients, CT-guided biopsy in $9 \%$, videoassisted thoracoscopic surgery (VATS) in 3\%, and mediastinoscopy in $2 \%$. Other procedures included diagnostic thoracotomy in 5\%, radical thoracic surgery with gain of histology in $1 \%$, ultrasonography (other than abdominal) $(1 \%)$ and extracranial MRI examinations. The frequencies of the procedures showed great variations between centres with particularly great variations for PET or PETCT (0-78\%), brain imaging (0-91\%) and EBUS/EUS (0-71\%) (Table 2).

Stage IIIA and stage IIIB were diagnosed in $55.1 \%$ and $44.9 \%$ of patients, respectively (Table 1 ). Subgroups based on $\mathrm{T}$ and $\mathrm{N}$ descriptors are shown in Table 3. Stages T1-T3 were found in 340 patients $(58.3 \%)$ mostly having N2 or N3 nodal stage in 301 (51.6\%) patients. T4N0, T4N1 and T4N2 tumours were found in 33 (5.7\%), 26 (4.5\%) and 129 (22.2\%) patients, respectively. T3N3 and T4N3 tumours were diagnosed in $29(5 \%)$ and $55(9.4 \%)$ patients, respectively. T3-4N3 tumours (stage IIIC according to $8^{\text {th }}$ TNM classification) were diagnosed in $84(14.4 \%)$ patients. N2 or N3 lymph nodes were found in $485(83.2 \%)$ patients. Histopathological involvement of mediastinal lymph nodes was confirmed in $172(29.5 \%)$ patients by surgery (96 patients), transbronchial biopsy (58 patients), medias- 
tinoscopy (11 patients) or other types of biopsy (10 patients) (data not shown).

\section{Treatment}

Therapeutic modalities are shown in overview for the total study population in Figure 1, for patients with stage IIIA and those with IIIB in detail in Table 4. Time from diagnosis to treatment initiation ranged from 0 to 369 (median 23) days. Combined therapies of any type were delivered to $326(55.9 \%)$ patients. With regard to a single treatment modality only, surgery was delivered to $1.5 \%$ thoracic radiotherapy to $11.8 \%$, chemotherapy to $25.7 \%$ and best supportive care (BSC) to $5.0 \%$ of patients of the total study population. Prophylactic cranial irradiation was not delivered to any patient.

Surgery was performed in 135 (23.2\%) patients (119 IIIA, 16 IIIB) and resulted in radical tumour resection in 121 patients and explorative surgery or non-radical resections in 14 patients (Table 4 and Figure 1). The types of surgery included lobectomy (77 patients), bi-lobectomies (6 patients), pneumonectomy (32 patients) and atypical radical resec-

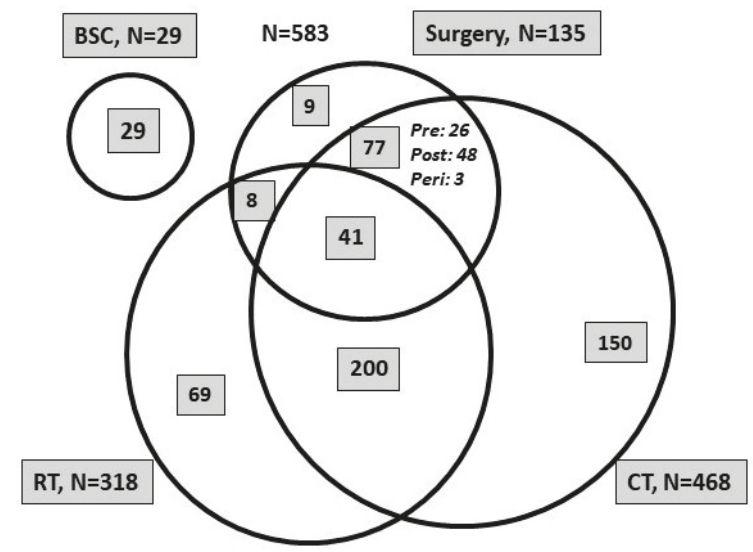

BSC = best supportive care; $C T$ = chemotherapy; pre = preoperative; post = postoperative; peri = perioperative; $\mathrm{RT}=$ radiotherapy

FIGURE 1. Treatment modalities and combinations.

tions (6 patients) (Table 4). Surgery was delivered as single modality (nine patients), in combination with chemotherapy (77 patients) or radiotherapy (eight patients) or as trimodality therapy (41 patients). Adjuvant chemotherapy was about twice as frequent as pre-operative chemotherapy among patients undergoing surgery.

TABLE 4. Therapeutic modalities and combinations

\begin{tabular}{|c|c|c|c|}
\hline Therapeutic modalities & IIIA (N=321) & $\begin{array}{l}\text { IIIB }(N=262) \\
N(\%)\end{array}$ & IIIA+IIIB (N=583) \\
\hline Any combination & $207(64.5)$ & $119(45.4)$ & $326(55.9)$ \\
\hline Surgery & $119(37.1)$ & $16(6.1)$ & $135(23.2)$ \\
\hline Surgery alone & $9(2.8)$ & 0 & $9(1.5)$ \\
\hline Surgery plus radiotherapy & $7(2.2)$ & $1(0.4)$ & $8(1.4)$ \\
\hline $\begin{array}{l}\text { Surgery plus chemotherapy } \\
\text { Preoperative chemotherapy } \\
\text { Perioperative chemotherapy } \\
\text { Adjuvant chemotherapy }\end{array}$ & $\begin{array}{l}70(21.8) \\
22(6.9) \\
3(0.9) \\
45(14.0)\end{array}$ & $\begin{array}{l}7(2.7) \\
4(1.5) \\
0 \\
3(1.1)\end{array}$ & $\begin{array}{l}77(13.2) \\
26(4.4) \\
3(0.5) \\
48(8.2)\end{array}$ \\
\hline $\begin{array}{l}\text { Surgery plus RT plus CT (trimodality) } \\
\text { Sequential preoperative RT plus CT } \\
\text { Concurrent preoperative RT plus CT } \\
\text { Sequential postoperative RT plus CT } \\
\text { Concurrent postoperative RT plus CT }\end{array}$ & $\begin{array}{c}33(10.3) \\
2(0.6) \\
3(0.9) \\
23(7.2) \\
5(1.6)\end{array}$ & $\begin{array}{l}8(3.1) \\
0 \\
1(0.4) \\
3(1.1) \\
4(1.5)\end{array}$ & $\begin{array}{l}41(7.0) \\
2(0.3) \\
4(0.7) \\
26(4.5) \\
9(1.5)\end{array}$ \\
\hline $\begin{array}{l}\text { Thoracic RT (including other modalities) } \\
\text { Radiotherapy alone }\end{array}$ & $\begin{array}{l}174(54.2) \\
37(11.5)\end{array}$ & $\begin{array}{l}144(55.0) \\
32(12.2)\end{array}$ & $\begin{array}{l}318(54.6) \\
69(11.8)\end{array}$ \\
\hline $\begin{array}{l}\text { Chemoradiotherapy } \\
\text { Sequential } \\
\text { Concurrent }\end{array}$ & $\begin{array}{l}97(30.2) \\
74(23.1) \\
23(7.2)\end{array}$ & $\begin{array}{l}103(39.3) \\
72(27.5) \\
31(11.8)\end{array}$ & $\begin{array}{c}200(34.3) \\
146(25.0) \\
54(9.3)\end{array}$ \\
\hline $\begin{array}{l}\text { Chemotherapy (including other modalities) } \\
\text { Chemotherapy alone }\end{array}$ & $\begin{array}{l}256(79.7) \\
58(18.1)\end{array}$ & $\begin{array}{l}212(80.9) \\
92(35.1)\end{array}$ & $\begin{array}{l}468(80.3) \\
150(25.7)\end{array}$ \\
\hline Best supportive care alone & $9(2.8)$ & $20(7.6)$ & $29(5.0)$ \\
\hline Type of surgery & $\operatorname{IIIA}(\mathrm{N}=119)$ & IIIB $(N=16)$ & IIIA+IIIB $(N=135)$ \\
\hline $\begin{array}{l}\text { Lobectomy } \\
\text { Bi-lobectomy } \\
\text { Pneumonectomy } \\
\text { Atypical radical resection } \\
\text { Non-radical surgery* }\end{array}$ & $\begin{aligned} 74 & (62.3) \\
6 & (5.0) \\
28 & (23.5) \\
5 & (4.2) \\
6 & (5.0)\end{aligned}$ & $\begin{array}{l}3(18.7) \\
0 \\
4(25.0) \\
1(6.2) \\
8(50.0)\end{array}$ & $\begin{aligned} 77 & (57.0) \\
6 & (4.5) \\
32 & (23.7) \\
6 & (4.5) \\
14 & (10.4)\end{aligned}$ \\
\hline
\end{tabular}

$*$ = Non-radical surgery $(n=14)$ as biopsy $(n=4$, all IIIB), exploration $(n=7,\|I A=5\| I I B=2$,$) or palliative resection (n=3,\|I A=1\| I B=2,) ; C T=$ chemotherapy; RT = radiotherapy 

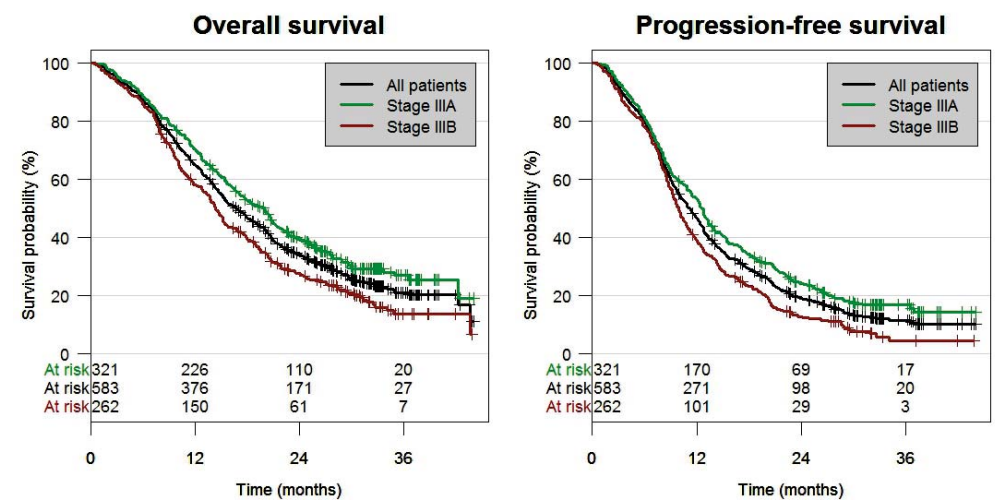

FIGURE 2. Overall survival and progression-free survival.

Thoracic radiotherapy was delivered to 318 (54.6\%) patients with similar proportions among stage IIIA and stage IIIB (Table 4). Thoracic radiotherapy was delivered as single modality (69 patients), as definitive chemoradiotherapy (200 patients) and as trimodality therapy (41 patients). Among patients undergoing definitive chemoradiotherapy, the sequential administration was much more frequent than the concurrent one (146 versus 54 patients). Among patients undergoing tri- modality therapy, concurrent chemoradiotherapy was delivered in 13 patients (Table 4).

Chemotherapy as the most frequent treatment modality was delivered to $468(80.3 \%)$ patients with similar percentages among IIIA and IIIB (Table 4). Chemotherapy was the only treatment modality in 150 patients. Carboplatin was used in $43.6 \%$ and cisplatin in $37.4 \%$ of patients (data not shown). Platins were combined with vinorelbine $(33 \%)$, gemcitabine $(22 \%)$, etoposide $(11 \%)$ or paclitaxel $(11 \%)$. Pemetrexed was used in $7.7 \%$ of patients in stage IIIB and $2.5 \%$ in stage IIIA. Other drugs including docetaxel, vinblastine and bevacizumab were each used in less than $2 \%$ of patients. No relevant differences in chemotherapy protocols between stage IIIA and IIIB patients were seen (data not shown).

\section{Treatment outcome}

At a median follow-up time of 30 months, 154 $(26.4 \%)$ patients were alive, $295(50.6 \%)$ patients were dead and $134(23 \%)$ patients were lost to follow-up. Among 334 patients evaluable for overall best response, complete remission, partial remis-
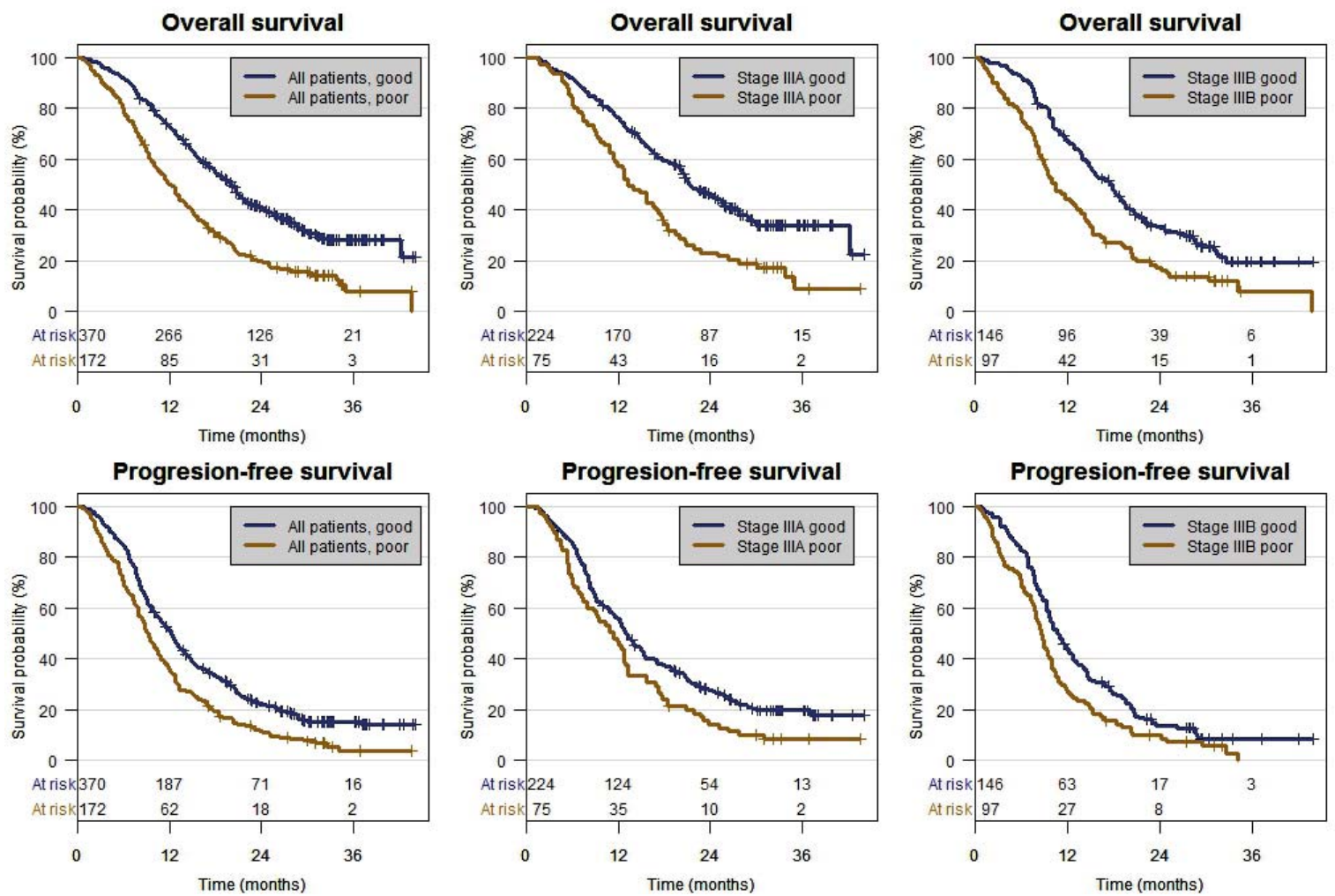

Good $=$ PS $0 / 1$ and no weight loss; poor $=$ PS $2 / 3$ and/or weight loss $\geq 10 \%$ within prior three months

FIGURE 3. Overall survival and progression-free survival by performance status and weight loss in all patients, stage IIIA and stage IIIB. 
TABLE 5. Overall survival according to stage and treatment modalities

\begin{tabular}{|c|c|c|c|c|c|}
\hline \multirow{2}{*}{ Characteristic } & N & Median & 1-year & 2-year & 3-year \\
\hline & & Months $(95 \% \mathrm{Cl})$ & \multicolumn{3}{|c|}{$\%(95 \% \mathrm{Cl})$} \\
\hline $\begin{array}{l}\text { IIIA } \\
\text { Total* } \\
\text { Good } \\
\text { Poor }\end{array}$ & $\begin{array}{l}321 \\
223 \\
75\end{array}$ & $\begin{array}{l}20.0(17.1 ; 21.4) \\
21.4(20.1 ; 25.8) \\
13.3(11.4 ; 17.7)\end{array}$ & $\begin{array}{l}70.7(65.9 ; 75.9) \\
76.3(70.9 ; 82.1) \\
57.3(47.2 ; 69.7\end{array}$ & $\begin{array}{l}39.4(34.3 ; 45.2) \\
45.6(39.4 ; 52.8) \\
23.2(15.2 ; 35.2)\end{array}$ & $\begin{array}{c}27.0(21.8 ; 33.3) \\
33.8(27.7 ; 41.3) \\
9.1(3.2 ; 26.0)\end{array}$ \\
\hline $\begin{array}{l}\text { IIIB } \\
\text { Total* } \\
\text { Good } \\
\text { Poor } \\
\text { T3N3 and T4N3** }\end{array}$ & $\begin{array}{c}262 \\
146 \\
97 \\
84\end{array}$ & $\begin{array}{l}14.4(13.0 ; 15.9) \\
17.8(14.8 ; 19.6) \\
10.4(8.8 ; 19.6) \\
11.6(9.8 ; 15.9)\end{array}$ & $\begin{array}{l}58.2(52.3 ; 64.5) \\
67.0(59.8 ; 75.1) \\
44.1(35.2 ; 55.2) \\
48.5(38.8 ; 60.5)\end{array}$ & $\begin{array}{l}27.5(22.5 ; 33.7) \\
33.1(26.1 ; 42.0) \\
17.3(11.1 ; 27.0) \\
22.6(15.2 ; 33.8)\end{array}$ & $\begin{array}{c}13.5(9.0 ; 20.2) \\
19.6(13.2 ; 29.1) \\
8.1(3.0 ; 21.6) \\
9.4(3.9 ; 22.7)\end{array}$ \\
\hline $\begin{array}{l}\text { IIIA+IIIB } \\
\text { Total* } \\
\text { Good } \\
\text { Poor }\end{array}$ & $\begin{array}{l}583 \\
369 \\
172\end{array}$ & $\begin{array}{l}16.8(15.3 ; 18.5) \\
20.1(18.0 ; 21.5) \\
11.8(10.2 ; 14.2)\end{array}$ & $\begin{array}{l}65.1(61.3 ; 69.1) \\
72.6(68.2 ; 77.3) \\
50.0(42.9 ; 57.9)\end{array}$ & $\begin{array}{l}34.1(30.4 ; 38.2) \\
40.7(35.9 ; 46.2) \\
19.9(14.6 ; 27.0)\end{array}$ & $\begin{array}{r}21.0(17.3 ; 25.4) \\
28.3(23.6 ; 34.0) \\
8.0(3.6 ; 17.8)\end{array}$ \\
\hline $\begin{array}{l}\text { Surgery } \\
\text { All surgeries } \\
\text { Alone } \\
\text { Surgery plus CT or RT } \\
\text { Surgery plus adjuvant CT } \\
\text { Trimodality therapy }\end{array}$ & $\begin{array}{c}135 \\
9 \\
85 \\
77 \\
41\end{array}$ & $\begin{array}{c}29.0(27.1 ; \mathrm{NA}) \\
13.9(3.2 ; \mathrm{NA}) \\
27.8(23.6 ; \mathrm{NA}) \\
28.3(24.7 ; \mathrm{NA}) \\
\text { Not reached }\end{array}$ & $\begin{array}{l}82.2(76.0 ; 88.9) \\
55.6(31.0 ; 99.7) \\
80.0(71.9 ; 89.0) \\
81.8(73.6 ; 90.9) \\
92.7(85.0 ; 100)\end{array}$ & $\begin{array}{l}60.6(52.8 ; 69.6) \\
22.2(6.6 ; 75.4) \\
57.2(47.5 ; 68.9) \\
60.6(50.6 ; 72.7) \\
76.9(64.7 ; 91.5)\end{array}$ & $\begin{array}{c}43.9(35.2 ; 54.8) \\
22.2(6.6 ; 75.4) \\
37.0(26.7 ; 51.3) \\
38.9(28.0 ; 54.0) \\
64.8(49.9 ; 84.2)\end{array}$ \\
\hline & & & $\mathrm{HR}(95 \% \mathrm{Cl})$ & p-value & \\
\hline IIIA vs. IIIB & & & $0.70(0.58 ; 0.85)$ & $<0.001$ & \\
\hline IIIA Good vs. IIIA Poor & & & $0.54(0.40 ; 0.73)$ & $<0.001$ & \\
\hline IIIB Good vs. IIIB Poor & & & $0.57(0.43 ; 0.76)$ & $<0.001$ & \\
\hline IIIA vs. IIIB Good & & & $0.86(0.68 ; 1.09)$ & 0.212 & \\
\hline Tri-modality vs. Surgery plus adjuvant CT & & & $0.47(0.25 ; 0.89)$ & 0.021 & \\
\hline
\end{tabular}

Good = performance status $0-1$ and no weight loss; Poor = performance status $2-3$ and/or weight loss $\geq 10 \%$; CRT = chemoradiotherapy; CT = chemotherapy; RT = radiotherapy * = Total is not sum of Good and Poor, as some patients had no data about weight loss;

** = Stage IIIC in Union for International Cancer Control [UICC] 8

sion, stable disease and progressive disease were seen in $27.5 \%, 35.9 \%, 24.9 \%$ and $11.7 \%$ patients, respectively. Information on progression during follow-up was available for 338 patients, while no information was available for the other patients due to death (171 patients) or loss to follow-up (74 patients). Eighty-two $(24.3 \%)$ patients were without progression and $256(75.7 \%)$ patients had progression, either local (40.2\%), distant (26.6\%) or both $(8.9 \%)$. Progression after end of treatment was more frequent among patients with stage IIIB than those with stage IIIA (91.2\% versus $81.6 \%$ ).

\section{Survival analyses}

OS and PFS are shown in Table 5, Table 6 and Figure 2. Median OS was 16.8 months and the 3-year OS rate was 21\%. Median PFS was 11.2 months and the 3-year PFS rate was $11.5 \%$. OS and
PFS were longer among patients with IIIA than among those with IIIB and the corresponding hazard ratios were $0.70(95 \%$ CI $0.58-0.85 ; \mathrm{p}<0.001)$ and 0.71 (95\% CI 0.59-0.85; p < 0.001), respectively (Table 5; Table 6). Among patients with stage IIIA, median OS was 20 months and the 3-year OS rate was $27 \%$. The corresponding values for patients with stage IIIB were 14.4 months and $13.5 \%$, respectively. Among patients with T3-4/N3 tumours who are classified as stage IIIC based on the $8^{\text {th }}$ edition of the TNM classification, survival outcome (median OS 11.6 months; 3-year OS rate 9.4\%) was worse than the outcome of patients with stage IIIB based on the $7^{\text {th }}$ TNM classification.

OS and PFS were longer among patients with PS 0-1 and no weight loss (good prognostic group) than among those with PS 2-3 and/or weight loss $\geq 10 \%$ (poor prognostic group) (Table 5; Table 6; Figure 3 ). These differences were observed in the 
TABLE 6. Progression free survival according to stage and risk groups

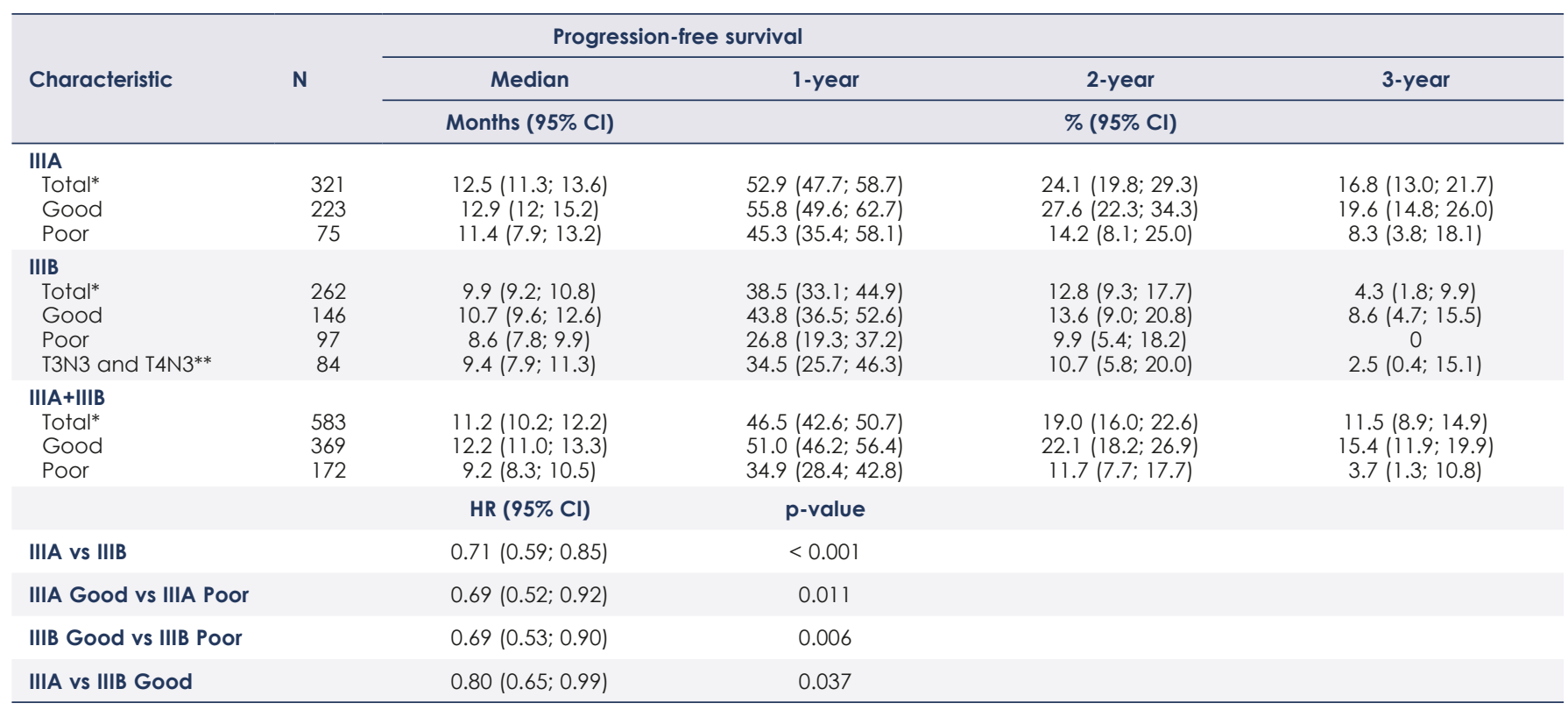

Good = performance status $0-1$ and no weight loss; Poor = performance status $2-3$ and/or weight loss $\geq 10 \%$;

* = Total is not sum of Good and Poor, as some patients had no data about weight loss:

** = Stage IIIC in Union for International Cancer Control [UICC] 8
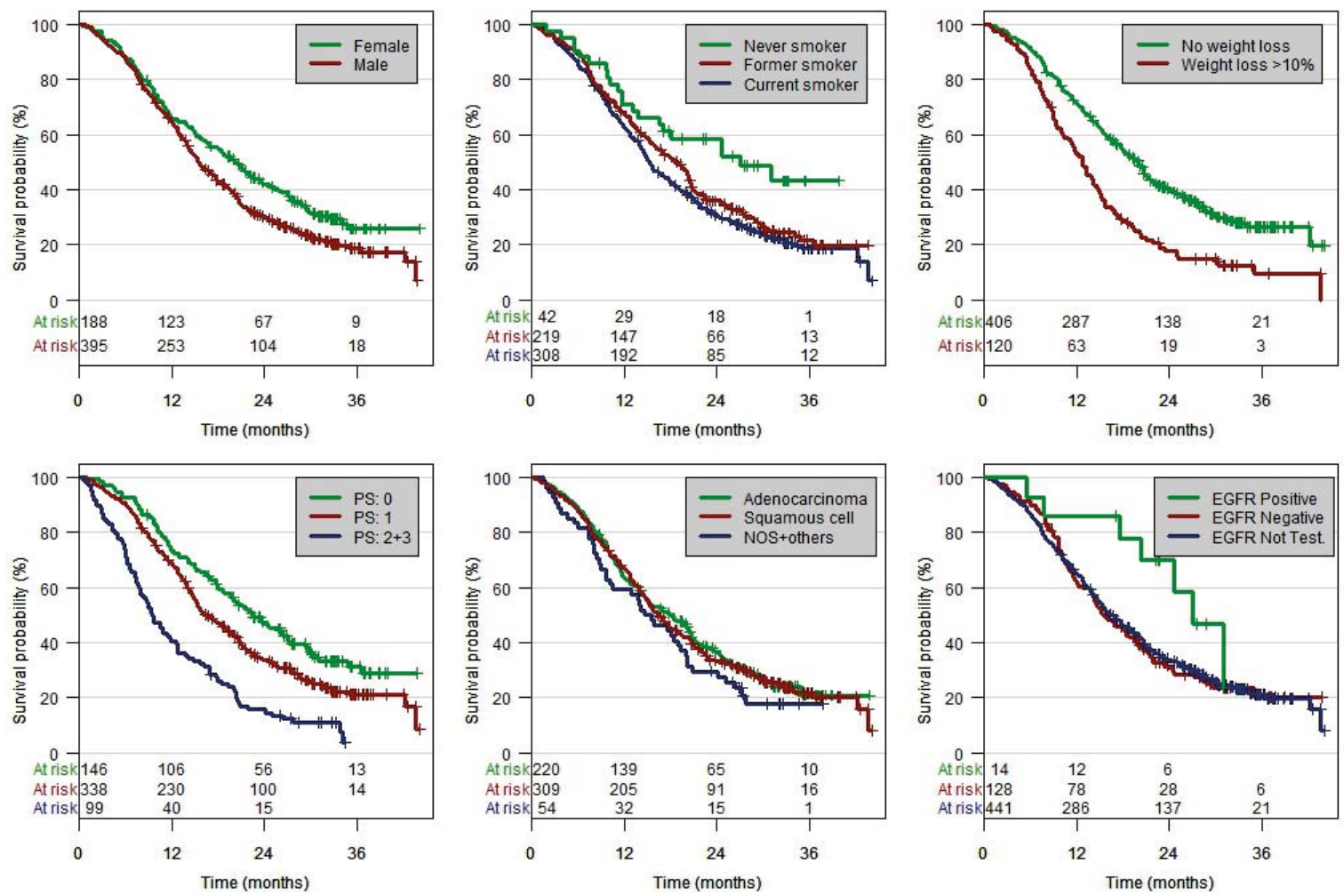

PS = performance status

FIGURE 4. Overall survival by variables. 
TABLE 7. Overall survival according to diagnostic variables

\begin{tabular}{|c|c|c|c|c|c|c|c|}
\hline \multirow{3}{*}{ Variable } & & \multirow{3}{*}{$\mathbf{N}$} & \multicolumn{5}{|c|}{ Survival } \\
\hline & & & \multirow{2}{*}{$\begin{array}{c}\text { Median } \\
\text { months }(95 \% \mathrm{Cl})\end{array}$} & Univariate analysis & \multirow{2}{*}{ P-value } & Multivariate analysis & \multirow{2}{*}{ P-value } \\
\hline & & & & HR ( $95 \%$ Wald Cl) & & HR ( $95 \%$ Wald Cl) & \\
\hline Gender & $\begin{array}{l}\text { Female } \\
\text { Male }\end{array}$ & $\begin{array}{l}188 \\
395\end{array}$ & $\begin{array}{l}20.4(16.8 ; 23.8) \\
15.4(14.3 ; 17.6)\end{array}$ & $0.76(0.62 ; 0.94)$ & $\begin{array}{l}\text { Female vs. } \\
\text { male: } \\
0.010\end{array}$ & $0.78(0.64 ; 0.96)$ & 0.044 \\
\hline $\begin{array}{l}\text { Weight loss } \\
\geq 10 \%\end{array}$ & $\begin{array}{l}\text { No } \\
\text { Yes }\end{array}$ & $\begin{array}{l}406 \\
120\end{array}$ & $\begin{array}{l}20.0(17.9 ; 21.1) \\
12.7(10.8 ; 14.8)\end{array}$ & $0.54(0.43 ; 0.68)$ & $\begin{array}{l}\text { No vs. yes: } \\
<0.001\end{array}$ & $0.68(0.53 ; 0.88)$ & 0.003 \\
\hline PS & $\begin{array}{c}0 \\
1 \\
\geq 2\end{array}$ & $\begin{array}{c}146 \\
338 \\
99\end{array}$ & $\begin{array}{l}22.4(17.7 ; 26.1) \\
15.4(13.9 ; 17.2) \\
11.6(8.8 ; 14.7)\end{array}$ & $\begin{array}{c}0 \text { vs. } \geq 2: 0.37(0.28 ; 0.50) \\
1 \text { vs. } \geq 2: 0.52(0.41 ; 0.66) \\
0 \text { vs. } 1: 0.72(0.57 ; 0.92)\end{array}$ & $\begin{array}{r}<0.001 \\
<0.001 \\
0.007\end{array}$ & $\begin{array}{l}0.62(0.43 ; 0.88) \\
0.69(0.52 ; 0.91) \\
0.90(0.68 ; 1.17)\end{array}$ & $\begin{array}{l}0.007 \\
0.009 \\
0.422\end{array}$ \\
\hline Histology & $\begin{array}{l}\text { Squamous } \\
\text { Adenoca } \\
\text { NOS/others }\end{array}$ & $\begin{array}{c}309 \\
220 \\
54\end{array}$ & $\begin{array}{l}16.3(15.0 ; 19.0) \\
18.1(14.7 ; 20.6) \\
15(10.1 ; 20.1)\end{array}$ & $\begin{array}{l}\text { Squam: } 0.96(0.79 ; 1.18) \\
\text { NOS/oth: } 0.79(0.57 ; 1.11)\end{array}$ & $\begin{array}{l}\text { Adeno vs.: } \\
0.726 \\
0.18\end{array}$ & $\begin{array}{l}\text { Squam: } 0.93(0.68 ; 1.26) \\
\text { NOS/oth:0.96(0.63;1.48) }\end{array}$ & $\begin{array}{l}0.630 \\
0.857\end{array}$ \\
\hline $\begin{array}{l}\text { Nodes } \\
\text { confirmed }\end{array}$ & $\begin{array}{l}\text { No } \\
\text { Yes }\end{array}$ & $\begin{array}{l}411 \\
172\end{array}$ & $\begin{array}{l}14.4(13.3 ; 15.9) \\
24.7(21.0 ; 28.8)\end{array}$ & $0.56(0.45 ; 0.70)$ & $\begin{array}{l}\text { Yes vs. no: } \\
<0.001\end{array}$ & $0.67(0.52 ; 0.87)$ & 0.002 \\
\hline $\begin{array}{l}\text { PET-CT or } \\
\text { PET scan }\end{array}$ & $\begin{array}{l}\text { Yes } \\
\text { No }\end{array}$ & $\begin{array}{l}163 \\
420\end{array}$ & $\begin{array}{l}19.6(17.8 ; 22.8) \\
15.4(14.1 ; 17.6)\end{array}$ & $0.73(0.58-0.91)$ & $\begin{array}{c}\text { Yes vs. no: } \\
0.005\end{array}$ & $0.84(0.66 ; 1.07)$ & 0.160 \\
\hline
\end{tabular}

NOS = not otherwise specified; oth = others; nodes confirmed = mediastinal lymph-nodes histologically confirmed; PS = performance status

total study population, in IIIA patients and in IIIB patients. Among the good prognostic group of IIIB patients, the survival outcome was similar to the survival outcome of IIIA patients.

Additional outcome data are summarized in Tables 5, 6 and 7 and Figures 3 and 4. Univariate analysis demonstrated longer OS for females, never-smokers, good PS, stage IIIA, mediastinal node verification, and for those with PET/CT or PET staging exam. In contrast, OS was not different between patients with adenocarcinomas and those with squamous cell carcinomas. Including these variables into the multivariate survival proportional hazard model, the differences in OS remained significant for females versus males, no weight loss versus weight loss, PS $0-1$ versus $2-3$, stage IIIA versus IIIB, and pathological verification of mediastinal nodes versus no verification. EGFR mutation status was not significant in the multivariate analysis but trend for better survival was found in the univariate model (Table 7). OS was also assessed in various treatment subgroups. Significant survival benefits were seen in patients undergoing surgery compared to patients without surgery (HR $=0.43 ; 95 \%$ CI $0.33-0.56$; p-value < 0.001) and in patients receiving combined treatment modalities compared to patients with a single treatment modality (Table 5, hazard ratios not shown). The best
OS outcome was shown for patients receiving trimodality therapy (Table 5). A significant OS difference between concurrent and sequential chemoradiotherapy could not be demonstrated. Time from diagnosis to initiation of treatment start had no impact on outcome (analysis by quartiles, $\mathrm{p}=0.585$ ).

\section{Discussion}

Our observational study demonstrated a large heterogeneity in both diagnostic procedures and treatment modalities among patients with locally advanced NSCLC in the real-world setting in Central European countries. The results were based on 583 patients from 16 cancer centres of seven countries. The patient characteristics of our study were slightly different from those of recent phase 3 tri$\mathrm{als}^{14,16}$, and these differences might be explained, at least partly, by higher smoking rates in Central European countries compared to other countries and the real-world nature of our study. In our study, two thirds were male, about half of the patients were older than 65 years and $7 \%$ were neversmokers. These rates are lower in terms of females and never-smokers than in the PROCLAIM trial ${ }^{16}$, but similar to those of the PACIFIC trial. ${ }^{14}$ The ma- 
jority of our patients (53\%) had squamous cell carcinomas and this frequency is slightly higher than the one $(47 \%)$ of the PACIFIC trial. ${ }^{14}$ The low rate (6\%) of NSCLC not otherwise specified confirms that accurate pathological examination is well established in Central European countries. Most patients $(83 \%$ ) had good (WHO 0-1) PS and 70\% had no weight loss prior to diagnosis. Tumour stages IIIA and IIIB were diagnosed in 55\% and $45 \%$ of patients, respectively, and these percentages are similar to those of the PACIFIC trial. ${ }^{14}$ Although not mandatory for patients with stage III NSCLC, molecular analyses were performed in selected patients. The EGFR mutation rate of $10 \%$ is consistent with the previously reported rate of $13.8 \%$ among patients with advanced NSCLC. ${ }^{17}$ The percentage of patients with ALK translocations (2\%) was in the lower range of stage IV patients ${ }^{18,19}$, but similar to another study. ${ }^{20} \mathrm{PD}-\mathrm{L} 1$ testing was not yet standard for patients with NSCLC stage at the time of our study.

Our real-world data can also be compared with those from other recent real-world studies. ${ }^{20-23}$ All studies were similar in terms of median age (around 65 years), good PS (75-86\%), never-smokers $(4-9 \%)$ and percentage of adenocarcinomas (around $40 \%$ ). In comparison to our study, the proportion of females were slightly higher in the American study $(44.8 \%)$ and the Canadian study $(46 \%) .^{20,22}$ The percentages of stage IIIA patients were similar between our and the American study but higher $(77 \%)$ in the Canadian study. ${ }^{20,22}$

A major goal of our study was to determine the diagnostic and staging procedures (Table 2). Computer tomography of the chest, supplemented by CT or ultrasonography of the upper abdomen, was performed in most patients. PET-CT (or PET) and brain imaging were performed in only $28 \%$ and $20 \%$ of patients, respectively. These percentages, however, greatly varied between centres and reached almost $100 \%$ in some centres (Table 2). In the Canadian real-world study, $58 \%$ of patients received a PET scan as part of staging and $74 \%$ had baseline brain imaging. ${ }^{22}$ Bronchoscopy was performed in $89 \%$ of patients, thereby being the most frequent intervention for obtaining tumour material for histopathological diagnosis. Only 13.5\% of patients had histopathological mediastinal nodal staging prior to therapy. The American and Canadian real-world studies did not provide data on histopathological assessment of mediastinal lymph nodes prior to treatment. ${ }^{20,22}$ In a retrospective analysis of 106 patients who had been treated with definitive radiotherapy (plus chemotherapy in most patients), $48.1 \%$ of patients had pathological confirmation of nodal disease. ${ }^{24}$

Treatment of our patients involved surgery, radiotherapy and chemotherapy (Table 4). Surgery with and without any other treatment was performed in $23 \%$ of all patients, $37.1 \%$ and $6.1 \%$ of patients with stage IIIA and IIIB, respectively. This percentage is similar to the Canadian study in which $21 \%$ of patients underwent surgery, either alone or as part of combined modality therapy. This similarity is somewhat surprising based on the fact that the percentage of our stage IIIB patients was higher than the Canadian one ( $45 \%$ versus $33 \%$ ). Lobectomy and pneumonectomy were performed in $57 \%$ and $24 \%$ of patients, respectively. The rates of pneumonectomies were similar between our and the Canadian study (23\% versus $18 \%$ ). Like in the Canadian study ${ }^{22}$, most of our surgical patients $(87 \%)$ received chemotherapy, more frequently as adjuvant than induction chemotherapy.

Chemoradiotherapy was delivered to $34.3 \%$ of our patients (Table 4). The concurrent approach was chosen only in $27 \%$ of these patients. This low frequency compared to other studies ${ }^{21,22}$ may be explained by patients unfit for the concurrent approach, limitations in the infrastructure of several centres including long waiting lists, and a stricter definition of concurrent versus sequential chemoradiotherapy than in other studies.

Overall, $80.3 \%$ of patients received systemic chemotherapy, either alone or combined with other treatment modalities. Radiotherapy alone and BSC alone were given to $11.8 \%$ and $5 \%$ of patients, respectively (Table 4). These percentages are in agreement with the percentage of patients with poor $(\mathrm{WHO} \geq 2)$ performance status. Although the proportion of patients with BSC was small and most likely caused by patient selection, it was similar to another study in which radiotherapy and BSC alone were delivered to $11 \%$ and $21 \%$ of patients, respectively. ${ }^{24}$

Our study confirmed tumour stage (IIIA versus IIIB), PS, prior weight loss and gender as prognostic factors. OS of patients with adenocarcinomas did not differ from the one of patients with squamous cell NSCLC. Patients undergoing surgery had better survival than those without surgery (Table 5). The combination of surgery with chemotherapy and/or radiotherapy was also associated with improved survival outcome. The lack of a survival benefit for our patients undergoing concurrent chemoradiotherapy compared to those undergoing sequential chemoradiotherapy in our real-world study (Table 5) might be explained by 
the multicentre nature of our study, low number of patients, and the heterogeneity of stage III NSCLC. The survival outcomes were similar between our study and the American real-world study..$^{20}$ Median OS times were 20.0 and 22.3 months for stage IIIA patients, respectively, and 14.4 and 14.7 months for stage IIIB patients, respectively. The longer median OS of 27.3 months in the Canadian study can, at least partly, be explained by the higher percentage of stage IIIA patients. The best OS in our study with $64 \%$ of patients being alive at three years was seen among patients undergoing trimodality therapy and was consistent with the Canadian study. ${ }^{22}$ It remains unclear, however, whether this survival benefit is due to treatment or just selection of patients with good prognostic features. In a randomized trial, the addition of surgery to chemoradiotherapy failed to improve overall survival. ${ }^{25}$

Our observational study has several limitations. Firstly, the findings were based on major academic cancer centres and, therefore, might not be representative for smaller centres. Secondly, there was no even distribution of patients between centres and an abundance of Czech patients was seen. Finally, other patient selection biases including patients lost to follow-up might have also played some role.

Our real-world findings also define areas for future improvements in the management of patients with locally advanced NSCLC in Central Europe. Firstly, the rates of staging by means of PET-CT must be increased, mainly through improving access to these procedures. Secondly, brain imaging should be implemented prior to treatment in all patients who are planned for aggressive treatments such as surgery or chemoradiotherapy. Thirdly, invasive staging of mediastinal lymph nodes prior to treatment should be performed more frequently. In order to achieve these goals, awareness among doctors has to be raised, opportunities for education as well as training of doctors must be increased, and the infrastructure of some centres has yet to be improved.

\section{Conclusions}

The present real-world study confirmed known prognostic factors and the broad heterogeneity in diagnostic and therapeutic strategies among patients with stage III NSCLC in Central European countries. Rates of PET-CT staging, invasive staging of mediastinal nodes, and brain imaging prior to combined treatments should be increased in the future.

\section{Acknowledgements}

We would like to thank the heads of the all participating centres for permission to use data of patients from their respective regional networks and for the support of this project. We are also indebted to all physicians who provided data for the registry.

This work was supported by the Czech Lung Cancer Cooperative Group (Kooperativní skupina pro léčbu plicní rakoviny, spolek).

\section{References}

1. Nappi A, Gallicchio R, Simeon V, Nardelli A, Pelagalli A, Zupa A, et al. [F-18] FDG-PET/CT parameters as predictors of outcome in inoperable NSCLC patients. Radiol Oncol 2015; 49: 320-6. doi: 10.1515/raon-2015-0043

2. Eberhardt WE, De Ruysscher D, Weder W, Le Péchoux C, De Leyn P, Hoffmann $\mathrm{H}$, et al. Panel members. 2nd ESMO consensus conference in lung cancer: locally advanced stage III non-small-cell lung cancer. Ann Oncol 2015; 26: 1573-88. doi: 10.1093/annonc/mdv187

3. Goldstraw P, Crowley J, Chansky K, Giroux DJ, Groome PA, Rami-Porta R, et al. The IASLC Lung Cancer Staging Project: proposals for the revision of the TNM stage groupings in the forthcoming (seventh) edition of the TNM classification of malignant tumours. J Thorac Oncol 2007; 2: 706-14. doi: 10.1097/JTO.0b013e31812f3c1a

4. Goldstraw P, Chansky K, Crowley J, Rami-Porta R, Asamura H, Eberhardt WE, et al. International Association for the Study of Lung Cancer Staging and Prognostic Factors Committee, Advisory Boards, and Participating Institutions. The IASLC Lung Cancer Staging Project: proposals for the revision of the TNM stage groupings in the forthcoming (eighth) edition of the TNM classification for lung cancer. J Thorac Oncol 2016; 11: 39-51. doi: 10.1016/j.jtho.2015.09.009.

5. Pignon JP, Tribodet H, Scagliotti GV, Douillard JY, Shepherd FA, Stephens $\mathrm{RJ}$, et al. Lung adjuvant cisplatin evaluation: a pooled analysis by the LACE collaborative group. J Clin Oncol 2008; 26: 3552-9. doi: 10.1200/ JCO.2007.13.9030

6. NSCLC Meta-analysis Collaborative Group. Preoperative chemotherapy for non-small-cell lung cancer: a systematic review and meta-analysis of individual participant data. Lancet 2014; 383: 1561-71. doi: 10.1016/S01406736(13)62159-5

7. Betticher DC, Hsu Schmitz SF, Totsch M, Hansen E, Joss C, von Briel C, et al. Prognostic factors affecting long-term outcomes in patients with resected stage IIIA pN2 non-small-cell lung cancer: 5-year follow-up of a phase II study. Br J Cancer 2006; 94: 1099-106.

8. Sher DJ, Fidler MJ, Liptay MJ, Koshy M. Comparative effectiveness of neoadjuvant chemoradiotherapy versus chemotherapy alone followed by surgery for patients with stage IIIA non-small cell lung cancer. Lung Cancer 2015; 88: 267-74. doi: 10.1016/j.lungcan.2015.03.015

9. Thomas M, Rube C, Hoffknecht P, Macha HN, Freitag L, Linder A, et al. Effect of preoperative chemoradiation in addition to preoperative chemotherapy: a randomised trial in stage III non-small-cell lung cancer. Lancet Oncol 2008; 9: 636-48. doi: 10.1016/S1470-2045(08)70156-6

10. Garrido P, Gonzalez-Larriba JL, Insa A, Provencio M, Torres A, Isla D, et al Long-term survival associated with complete resection after induction chemotherapy in stage IIIA (N2) and IIIB (T4NO-1) non-small-cell lung cancer patients: the Spanish Lung Cancer Group Trial 9901. J Clin Oncol 2007; 25: 4736-42. 
11. Barlési F, Doddoli C, Torre JP, Giudicelli R, Fuentes P, Thomas P, et al. Comparative prognostic features of stage IIIAN2 and IIIB non-small-cell lung cancer patients treated with surgery after induction therapy. Eur $J$ Cardiothorac Surg 2005; 28: 629-34. doi: 10.1016/j.ejcts.2005.06.018

12. Auperin A, Le Pechoux C, Rolland E, Curran WJ, Furuse K, Fournel P, et al. Meta-analysis of concomitant versus sequential radiochemotherapy in locally advanced non-small-cell lung cancer. J Clin Oncol 2010; 28: 2181-90. doi: $10.1200 / J C O .2009 .26 .2543$

13. Ma L, Men Y, Feng L, Kang J, Sun X, Yuan M, et al. A current review of doseescalated radiotherapy in locally advanced non-small cell lung cancer. Radio Oncol 2019; 53: 6-14. doi: 10.2478/raon-2019-0006

14. Antonia SJ, Villegas A, Daniel D, Vicente D, Murakami S, Hui R, et al PACIFIC Investigators. Durvalumab after chemoradiotherapy in stage II non-small-cell lung cancer. N Engl J Med 2017; 377: 1919-29. doi: 10.1056/ NEJMoa1709937

15. Zhang Z, Sun J. Interval censoring. Stat Methods Med Res 2010; 19: 53-70. doi: $10.1177 / 0962280209105023$

16. Senan S, Brade A, Wang LH, Vansteenkiste J, Dakhil S, Biesma B, et al. PROCLAIM: Randomized phase III trial of pemetrexed-cisplatin or etoposide-cisplatin plus thoracic radiation therapy followed by consolidation chemotherapy in locally advanced nonsquamous non-small-cell lung cancer. J Clin Oncol 2016; 34: 953-62. doi: 10.1200/JCO.2015.64.8824

17. Ramlau R, Cufer T, Berzinec P, Dziadziuszko R, Olszewski W, Popper H, et al. INSIGHT study team. Epidermal growth factor receptor mutation-positive non-small-cell lung cancer in the real-world setting in Central Europe: The INSIGHT Study. 10: 1370-4. J Thorac Oncol 2015; 10: 1370-4. doi: 10.1097/ JT0.0000000000000621

18. Barlesi F, Mazieres J, Merlio JP, Debieuvre D, Mosser J, Lena $H$, et al. Biomarkers France contributors. Routine molecular profiling of patients with advanced non-small-cell lung cancer: results of a 1-year nationwide programme of the French Cooperative Thoracic Intergroup (IFCT). Lancet 2016; 387: 1415-26. doi: 10.1016/S0140-6736(16)00004-0

19. Hofman P. ALK in Non-Small Cell Lung Cancer (NSCLC): pathobiology, epidemiology, detection from tumor tissue and algorithm diagnosis in a daily practice. Cancers 2017; 9: pii: E107. doi: 10.3390/cancers9080107

20. Ryan KJ, Skinner KE, Fernandes AW, Punekar RS, Pavilack M, Walker MS, et al. Real-world outcomes in patients with unresected stage III non-small cell lung cancer. Med Oncol 2019; 36: 24. doi: 10.1007/s12032-019-1249-1

21. Ryan KJ, Skinner KE, Fernandes AW, Punekar RS, Pavilack M, Walker MS, et al. Real-world treatment patterns among patients with unresected stage III non-small-cell lung cancer. Future Oncol 2019; 15: 2943-53. doi: 10.2217/ fon-2018-0939

22. Moore S, Leung B, Wu J, Ho C. Real-world treatment of stage III NSCLC: the role of trimodality treatment in the era of immunotherapy. $J$ Thorac Oncol 2019; 14: 1430-9. doi: 10.1016/j.jtho.2019.04.005

23. Fernandes AT, Mitra N, Xanthopoulos E, Evans T, Stevenson J, Langer C, et al. The impact of extent and location of mediastinal lymph node involvement on survival in stage III non-small cell lung cancer patients treated with definitive radiotherapy. Int J Radiat Oncol Biol Phys 2012; 83: 340-7. doi: 10.1016/j.jijrobp.2011.05.070

24. Van der Meer FS, Schramel FM, Van Vulpen M, El Sharouni SY. Feasibility of concomitant chemoradiotherapy in daily practice for patients with NSCLC stage III. Anticancer Res 2016; 36: 4673-6. doi: 10.21873/anticanres.11019

25. Albain KS, Swann RS, Rusch VW, Turrisi AT 3rd, Shepherd FA, Smith C, et al. Radiotherapy plus chemotherapy with or without surgical resection for stage III non-small-cell lung cancer: a phase III randomised controlled trial. Lancet 2009; 374: 379-86. doi: 10.1016/S0140-6736(09)60737-6 